\title{
Modal cut-on ratio in ducts with realistic flow profiles and its application to acoustic mode detection
}

\author{
J. R. Mathews ${ }^{\mathrm{a}}$, J. Chen ${ }^{\mathrm{b}}$, P. Joseph ${ }^{\mathrm{b}, *}$ \\ ${ }^{a}$ Whittle Laboratory, Department of Engineering, University of Cambridge, Cambridge. \\ ${ }^{b}$ Institute of Sound and Vibration, University of Southampton
}

\begin{abstract}
This paper considers the modal cut-on ratio and its use in acoustic mode detection in ducts for a radially varying flow with swirl. These concepts have previously only been considered for the idealised case of uniform axial mean flow and no swirl, and thus in this paper they are generalised and extended to account for radial variations in axial and swirl Mach numbers. The cut-on ratio is shown to no longer satisfy a simple relation with the axial wavenumber, showing that while the cut-on ratio in arbitrary flow is defined its usefulness significantly decreases. Mode distribution functions are also investigated for radially varying flows, both in terms of cut-on ratio and axial wavenumber. In view of the deficiencies of the cut-on ratio highlighted in the paper a recent method for mode detection in ducts that uses just two microphones is generalised to deduce the mode amplitudes in radially varying flows, based on modal axial wavenumber rather than cut-on ratio. This method is shown to perform better than the existing method in radially varying and/or swirling flow in simple test configurations with an idealised source distribution.
\end{abstract}

Keywords: Cut-on ratio, cut-on frequency, mode detection, two-microphone method

\section{Introduction}

This paper deals with the definition of modal cut-on (or cut-off) ratio in ducts with realistic mean flow profiles and its application to mode detection in high frequency broadband sound fields. The concept of cut-on ratio was proposed in the early 1970's by (author?) [1] as a non-dimensional measure of the extent to which an acoustic mode in a duct is cut-on. In approximate terms it is related to how high in frequency $\omega$ an acoustic mode is excited above its cut-on, or resonance, frequency $\omega_{m n}$. A mode excited at a single frequency well above its cut-on frequency, $\omega \gg \omega_{m n}$, is said to be well cut-on and its wavefront normal vector propagates at a shallow angle to the duct axis. At frequencies close to the cut-on frequency, the mode is said to be close to cut-off, whose modal wavefront normal vector propagates at a large angle to the duct axis. These modes transport comparatively little sound power along the duct, tending to zero at cut-off. The usefulness of this concept is that in a hard-walled duct containing a uniform mean axial flow, different modes with different values of mode indices $(m, n)$, but with the same cut-on ratio, have similar transmission characteristics along the duct and similar radiation characteristics to the far field, as indicated in Section 1.2 below.

The concept of modal cut-on (or cut-off ratio) is now is common usage in duct acoustics $[2,3,4,5]$ as a single index of the degree to which a single mode is cut-on. It is a well-defined quantity in the idealised case of a hard-walled duct containing a uniform mean axial flow and can be used to deduce the modal propagation angle within the duct [6] and also the angle of the main lobe upon radiation to the far field $[7,8]$. However, its definition in realistic mean flow profiles, and more importantly its interpretation, is less clear. These issues are addressed in this paper.

\footnotetext{
* Corresponding author

Email addresses: jrm214@cam.ac.uk (J. R. Mathews), jc2d14@soton.ac.uk (J. Chen), pfj@isvr.soton.ac.uk (P. Joseph)
} 
We start by reviewing the basic relations between cut-on ratio and modal wavenumbers for the idealised case of uniform mean axial flow. These are generalised to arbitrary mean flow profiles including the effects of swirl, whose usefulness and limitations are then explored. Finally, its application to the new two-microphone method recently proposed by (author?) [9] is investigated in which the concept of cut-on ratio and its simple relationship to the modal axial wavenumber is central. Here we show that this method, which aims to determine the distribution of mode amplitudes versus cut-on ratio, breaks down. An alternative formulation is proposed that overcomes these issues.

\subsection{Modal cut-on Ratio}

The usefulness of the cut-on ratio was first proposed by (author?) [10, 11]. Rice's innovation was to recognise that in a hard-walled duct containing a uniform mean axial flow, different modes with different values of mode indices $(m, n)$, but with the same cut-on ratio $\alpha$, had similar transmission characteristics along the duct and similar radiation characteristics to the far field. In particular Rice showed that for this idealised case, cut-on ratio was uniquely and simply related to the angle at which the modal wavefront normal vector makes with the duct axis, which was also equal to the angle of the main far-field radiation lobe, for the idealised case in which the flow speed was everywhere the same. Rice used this concept to derive mode amplitude distributions that vary smoothly with cut-on ratio for some idealised source distributions, such as when each mode is assumed to contain identical sound power [1]. (author?) [12] later extended this concept to express the amplitude distribution of a broader family of physically important source distributions in terms of $\alpha$.

We start by reviewing the basic relations between modal cut-on ratio $\alpha$ and the modal wavenumber components in a cylindrical hard-walled hollow or annular duct (with hub-to-tip ratio $h$ ) containing a uniform axial flow. The outer duct wall has been non-dimensionalised so that $r=1$. At a single non dimensional frequency $\omega$ the acoustic pressure propagating along the duct is of the form,

$$
p(r, \theta, x)=e^{-\mathrm{i} \omega t} \sum_{m=-\infty}^{\infty} \sum_{n=0}^{\infty} A_{m n}^{ \pm} P_{m n}^{ \pm}(r) e^{\mathrm{i} m \theta} e^{\mathrm{i} \omega k_{m n}^{ \pm} x},
$$

where $A_{m n}^{ \pm}$is the pressure mode amplitude, $m$ is the azimuthal mode order, $P_{m n}^{ \pm}(r)$ is the normalised, nondimensional mode shape over the duct cross section and $k_{m n}$ is the non-dimensional axial wavenumber, which specifies the rate of change of phase with axial distance $x$ along the duct for each mode. The superscripts \pm correspond to downstream modes $(+)$ and upstream modes $(-)$.

Substituting (1) into the convected wave equation with the axial Mach number $M_{x}$ assumed to be constant over the duct cross section yields the expression for $k_{m n}$ of the form,

$$
k_{m n}=\frac{\alpha_{m n}-M_{x}}{\beta^{2} c_{0}},
$$

where $\beta^{2}=1-M_{x}^{2}$ and $c_{0}$ is the sound speed in the quiescent medium. This simple relationship between $k$ and $\alpha$ is central to the usefulness of the cut-on ratio as an index of modal behaviour. The relation in (2) makes explicit that the cut-on ratio $\alpha$ is simply the axial wavenumber $k$ scaled by $\beta^{2} c_{0}$ and shifted by $M_{x} \beta^{2} / c_{0}$, which explains the usefulness of $\alpha$. Any departure from this linear relationship leads to a loss in the usefulness of the cut-on ratio and ambiguity in its interpretation.

We non-dimensionalise velocities such that $c_{0}(1)=1$, so that for a uniform mean flow $c_{0}$ is identically unity and therefore doesn't explictely appear in (2). The quantity $\alpha_{m n}$ may be expressed in terms of the transverse modal wavenumber $\kappa_{m n}[13]$ :

$$
\alpha_{m n}^{ \pm}= \pm \sqrt{1-\beta^{2} \frac{\kappa_{m n}^{2}}{\omega^{2}}}
$$

For the case of uniform axial flow in a hollow duct, $\kappa_{m n}$ satisfies $J_{m}^{\prime}\left(\kappa_{m n}\right)=0$ and hence may be determined from tables of the stationary values of the Bessel function $J_{m}$, as given in (author?) [14]. We can also 
determine the axial wavenumbers $k_{m n}$ for uniform axial flow by solving the eigenvalue problem given in Appendix A (which also applies for an arbitrary swirling flow).

Equations 1 and 2 make explicit the existence of a frequency $\omega=\omega_{m n}$ at which $\alpha_{m n}^{ \pm}=0$, below which the mode decays exponentially along the duct away from the source and may be said to be cut-off. Setting $\alpha_{m n}^{ \pm}=0$ in (3) gives the cut-on frequency as $\omega_{m n}=\beta \kappa_{m n}$. At any arbitrary frequency $\omega$ therefore $\alpha_{m n}$ may be written as,

$$
\alpha_{m n}= \pm \sqrt{1-\frac{\omega_{m n}^{2}}{\omega^{2}}} .
$$

It therefore takes purely imaginary values for cut-off modes, is zero at cut-off and ranges between zero and unity as the frequency increases above cut-off. Other variants of this quantity are often used, such as the cut-off ratio $[6]$

$$
\xi_{m n}=\frac{1}{1-\alpha_{m n}^{2}}=\frac{\omega^{2}}{\omega_{m n}^{2}},
$$

which has the advantage of being real for both cut-on and cut-off modes.

This relationship has recently been used in a new measurement method by which the mode amplitude distribution versus $\alpha_{m n}$ can be determined from the cross spectrum of acoustic pressures between two closely spaced microphones at the duct wall. This method will be considered again in Sections 3 and 4 and shown to be inaccurate for realistic mean flow profiles due to the deficiencies of cut-on ratio in these cases.

\subsection{Modal propagation angles}

Before proceeding to investigate the meaning and interpretation of cut-on ratio for realistic mean flow profiles we first discuss its usefulness in determining the main modal propagation angles in uniform axial flow. Figure 1 show the modal wavefront, approximated as a plane wave, in the $r-x$ plane of the duct.

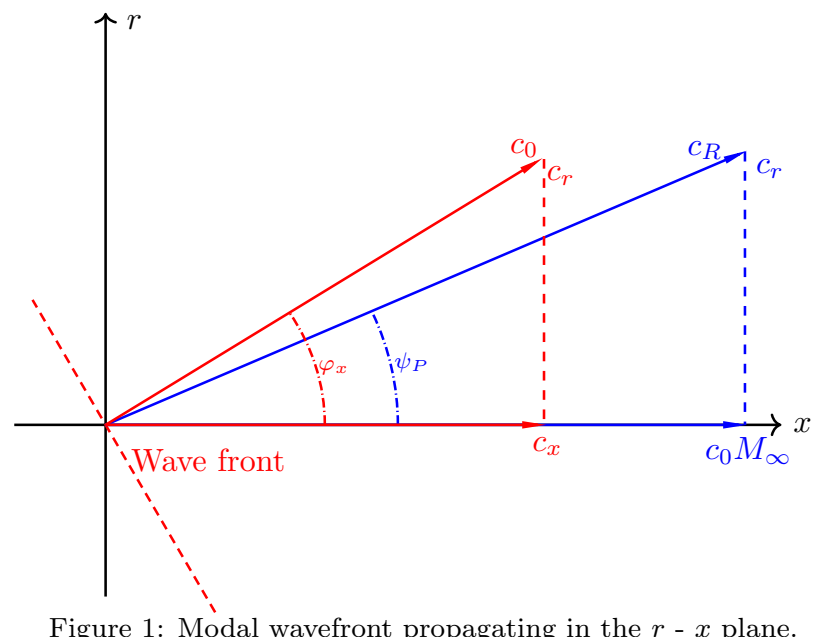

The normal to the modal wavefront propagates at the speed of sound $c_{0}$ at an angle $\varphi_{x}$ to the duct axis with components $\left(c_{x}, c_{r}\right)$, whose wavenumber components are in the same direction with magnitudes $k=\omega / c_{0}, k_{x}=\omega / c_{x}$ and $k_{r}=\omega / c_{r}$. However, in the presence of a uniform axial flow the resultant direction of propagation is $c_{R}$ with components $\left(c_{x}+c_{0} M_{x}, c_{r}\right)$ at an angle $\psi_{P}$ to the duct axis since the axial propagation speed includes an additional drift correction $c_{0} M_{x}$ corresponding to the axial flow speed. Making use of (2) and the geometry sketched in Figure 1, the two modal propagation angles $\varphi_{x}$ and $\psi_{P}$ may be expressed solely in terms of cut-on ratio or axial wavenumber as follows [6],

$$
\begin{aligned}
\cos \varphi_{x} & =\frac{k_{m n}}{1-k_{m n} M_{x}}=\frac{\alpha_{m n}-M_{x}}{1-\alpha_{m n} M_{x}}, \\
\cos \psi_{P} & =\frac{M_{x}+k_{m n} \beta^{2}}{\left[\left(1-k_{m n} M_{x}\right)\left(1+M_{x}\left(M_{x}+k_{m n} \beta^{2}\right)\right)\right]^{1 / 2}}=\frac{\alpha_{m n} \beta}{\left(1-\alpha_{m n}^{2} M_{x}^{2}\right)^{1 / 2}} .
\end{aligned}
$$


The two angles $\varphi_{x}$ and $\psi_{P}$ define the behaviour of modal propagation in the duct and to the far field. The first angle relates to the angle of the wavefront within the duct, and is linked to the phase speed of the mode, which is $\cos ^{-1}\left(M_{x}\right)$ at cut-on, tending to $0^{\circ}$ as the frequency is increased. The second angle $\psi_{P}$ quantifies the resultant propagation angle and is identical to the angle of the main radiation lobe as it radiates from the duct open end. At the cut-on frequency it takes the value of $90^{\circ}$ tending towards $0^{\circ}$ as the frequency is increased above the cut-on frequency. Clearly, therefore, different modes with different cut-on ratios have similar transmission and radiation characteristics for this idealised flow profile. It is also clear that the expression for the radiation angles are much simpler in terms of cut-on ratio than axial wavenumber.

\section{Defining the cut-on ratio for swirling flow in an annular duct}

As discussed in Section 1 above, the concept of cut-on ratio is well defined for a duct containing a uniform axial mean flow. Moreover, its relationship to the modal wavenumber components, and hence propagation angles, is straightforward. In the more realistic case of an arbitrary swirling flow, its definition, and usefulness, is less obvious. In this section we generalise the definition of the cut-on ratio $\alpha$ to include these general profiles and investigate its relationship to the axial wavenumber component $k_{m n}$, which as we saw in (6) is inextricably linked to the modal propagation angles $\varphi_{x}$ and $\psi_{P}$.

We now consider modal propagation in a hard-walled annular duct $h<r<1$ containing radially varying axial and circumferential velocity components $M_{x}(r)$ and $M_{\theta}(r)$, respectively.

\subsection{Defining the cut-on ratio for arbitrary swirling flow}

In this paper we define the cut-on ratio for arbitrary swirling flows to be

$$
\alpha_{m n}= \pm \sqrt{1-\omega_{m n}^{2} / \omega^{2}}
$$

where $\omega_{m n}$ is the cut-on frequency of the $(m, n)$-th mode (i.e. the frequency at which $k_{m n}$, goes from being cut-on $\left(\Im\left(k_{m n}\right)=0\right)$ to being cut-off $\left.\left(\Im\left(k_{m n}\right) \neq 0\right)\right)$. Note that this definition was chosen to have the desired property of spanning the range $[-1,1]$ for the range of propagating duct modes. We emphasise, however, that the choice of definition is essentially arbitrary. In choosing it to be defined by (7) it has the advantages of being entirely consistent with the case of uniform mean axial flow and, as we shall show below in Section 2.3 , has an identical distribution to that of a uniform axial flow even for large radial variations in axial and circumferential flow speeds.

In the general case the cut-off frequencies $\omega_{m n}$ are required to be calculated numerically, and a procedure for doing this is described in Appendix B.

\subsection{Relating the cut-on ratio and axial wavenumber}

In this section, the computed values of $\alpha$ deduced from the numerical procedure outlined in Appendix B are plotted against their corresponding values of $k$ (also calculated used the method described in Appendix A) for the three representative cases of: uniform axial flow, radially varying axial flow and radially varying swirling flow. These relationships will be shown to be key in establishing the validity of the theoretical linear relationship expressed in (2) in these cases, and hence establish the usefulness of cut-on ratio as a parameter for uniquely determining modal propagation.

We will see that the relationship between $\alpha$ and $k$ becomes significantly more complicated and nonlinear when radial variations to the flow are present. In these more realistic cases, therefore, cut-on ratio may not be as useful as a parameter for predicting modal propagation and an alternative parameter must be considered, as discussed in Section 3.

\subsubsection{Uniform axial flow}

Before discussing more realistic flow profiles, we first consider the idealised case of a uniform axial flow with Mach number $M_{x}=0.5$ in an annular duct with hub-to-tip ratio $h=0.5$ at a non-dimensional frequency of $\omega=20$. This case allows the validation of the numerical procedure aimed at reproducing the theoretical relation of (2). Figure 2 is a plot of $k$ against $\alpha$ (calculated using the cut-on frequency in (7)). Also shown is the dashed line of (2). Extremely good agreement is observed, thereby validating the numerical procedure. 


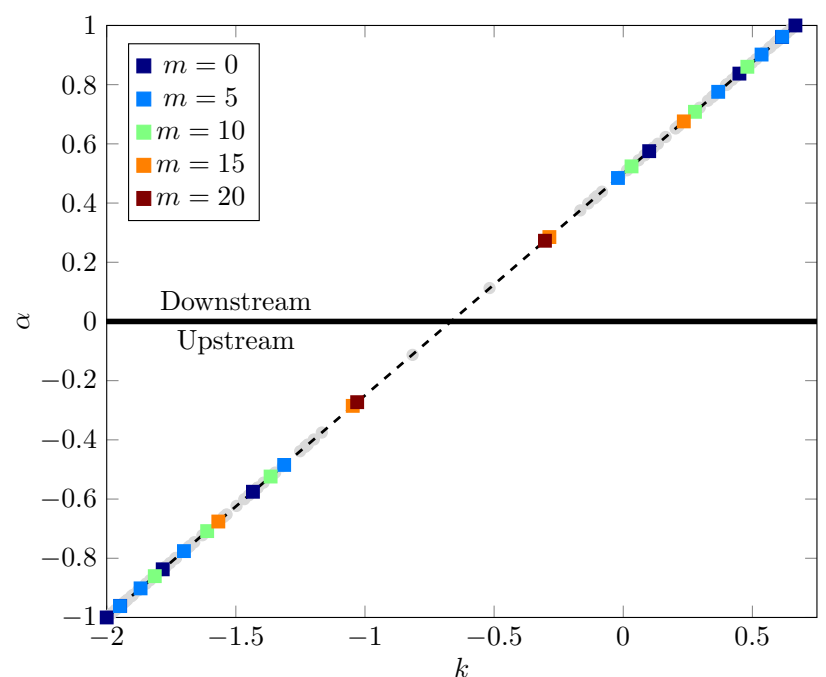

Figure 2: Plot of $k$ verses $\alpha$ in grey circles for $M_{x}=0.5, \omega=20, h=0.5$ and all values of $m$. Selected values of $m$ are given in coloured squares.

\subsubsection{Radially varying axial flow}

In this section the relationship between $\alpha$ and $k$ is investigated for the case of radially varying axial flow in which swirl is absent. Figures 3a, 3b and 3c are plots of $\alpha$ versus $k$ for the three idealised profiles of $M_{x}(r)=0.4+0.4(r-h), M_{x}(r)=0.33+0.66(r-h)$ and $M_{x}(r)=0.25+(r-h)$ at a non-dimensional frequency of $\omega=20$ in a duct with a hub-to-tip ratio of $h=0.5$. These shear flows profiles are designed to have increasing gradient but have an identical radially-averaged mean flow speed of $\overline{M_{x}}=0.5$. Also shown in these figures, as a dashed line, is the theoretical line of (2) showing the relationship for the case of uniform axial mean flow.

These figures demonstrate that the relationship $k(\alpha)$ is no longer linear and whose deviation from the uniform flow case (the black dashed line) increases as the shear flow gradient increases. In these figures the modal behaviour associated with some representative circumferential mode orders $m$ are indicated as coloured squares. It is clear from the figure that in these representative flow profiles, the behaviour of $k(\alpha)$ for individual modes is complicated and may be summarised as follows:

1. The relationship $k(\alpha)$ is monotonic for modes at a fixed value of $m$.

2. Modes of the same radial index $n$ (with $n=1$ corresponding to the most cut-on mode) but different $m$ values follow the same trajectory, which in this case appears to be approximately linear.

3. The variation of $\alpha$ for a given value of $k$ increases as $\alpha$ decreases from 1 to -1 . Greater variations in

(a)

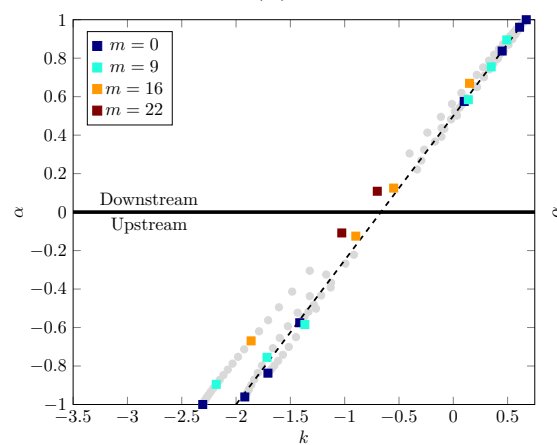

(b)

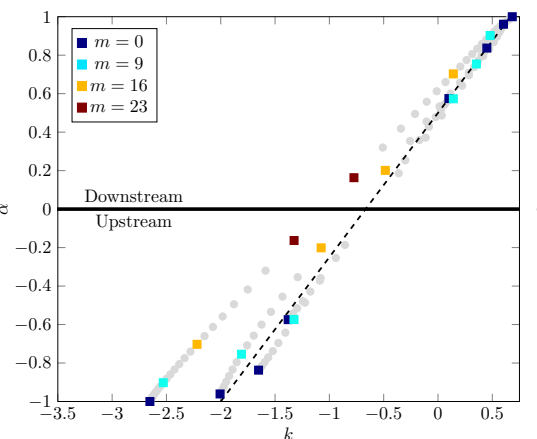

(c)

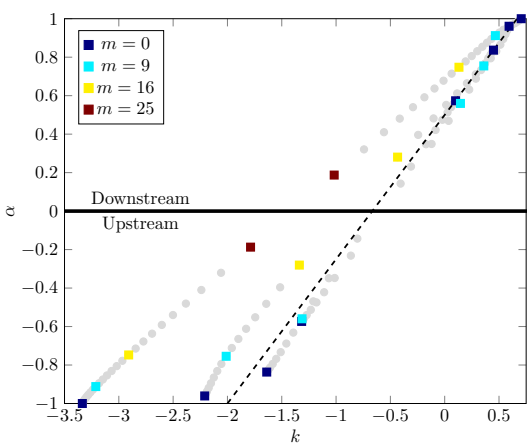

Figure 3: Plot of $k$ versus $\alpha$ for three different axial flows (grey circles), (a): $M_{x}(r)=0.4+0.4(r-h),(\mathrm{b})$ :

$M_{x}(r)=0.33+0.66(r-h)$ and $(\mathrm{c}): M_{x}(r)=0.25+(r-h)$. The other parameters are $h=0.5$ and $\omega=20$. Selected values of $m$ have been highlighted with coloured squares. In black (dashed) is the relationship between $k$ and $\alpha$ for uniform flow $\overline{M_{x}}=0.5$. 
(a)

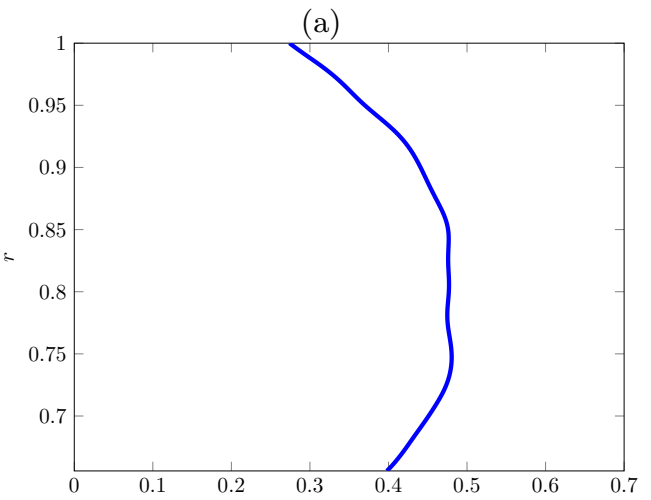

(b)

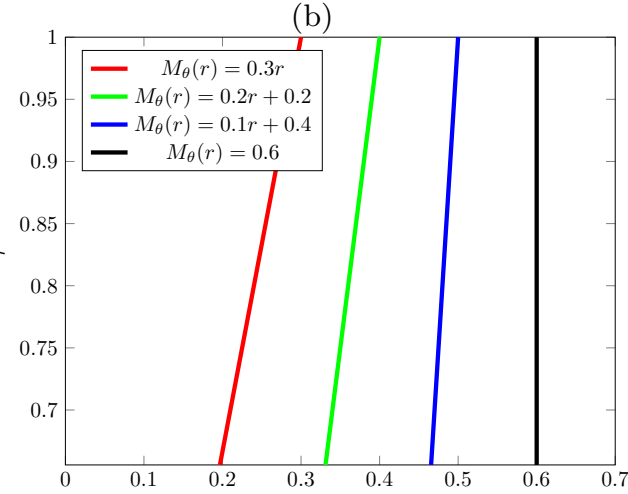

Figure 4: Plot of (a) axial flow $M_{x}(r)$ based on (author?) [15]. The hub-to-tip ratio is $h=0.6558$. In (b) we give the idealised swirling flows $M_{\theta}(r)$ that are considered later in Section 2.2.3.

$\alpha$ are predicted for upstream-going modes than downstream-going modes.

4. As a consequence of the second observation the relationship $k(\alpha)$ is no longer one-to-one, in the sense that a single value of $k$ can correspond to multiple values of $\alpha$. In particular, in Figure $3 \mathrm{c}, k \approx-1$, for example, could correspond to an upstream or a downstream mode, which are marked in the figure.

We can therefore conclude from Figure 3 that for small variations in the mean flow profile (such as in Figure 3a), the cut-on ratio remains a useful concept in determining the modal axial wavenumber $k$ and hence modal propagation, as sketched in Figure 1. However, for larger variations in flow profile (such as Figures 3b and 3c), $\alpha$ no longer accurately quantifies the behaviour of $k$ and hence modal propagation.

We now consider the relationship between $k$ and $\alpha$ for a more realistic axial flow profile. This profile was measured in the by-pass section of a scale-model aero-engine with hub-to-tip ratio of $h=0.6558$ in the Anecom test facility in Germany [15]. This profile is plotted in Figure 4a. The relationship between $\alpha$ and $k$ is plotted in Figure 5 at the two frequencies of $\omega=20$ and $\omega=80$. While all four features above are present in the plots, for this more realistic case the variation $k(\alpha)$ is much closer to the uniform profile case. The definition of cut-on ratio remains valid and useful for this more realistic non-swirling profile.

From Figure 5, it is clear that the relationship between $k$ and $\alpha$ are broadly similar at the low and high frequencies under consideration. Since calculating the wavenumbers $k$ and cut-on ratio $\alpha$ is more expensive at higher frequencies, we can thus examine the behaviour $k(\alpha)$ at low frequencies to determine the relationship between $k$ and $\alpha$, and the usefulness of the cut-on ratio.

At the present time we are unable to predict the extent of deviation of $k(\alpha)$ for any arbitrary flow profile

(a)

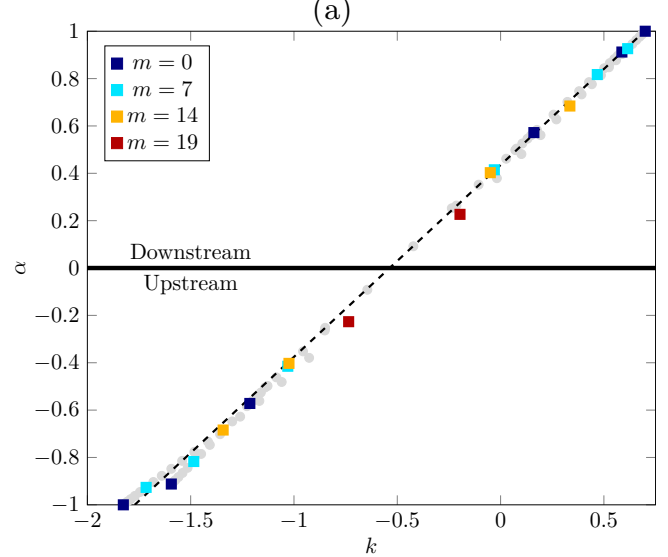

(b)

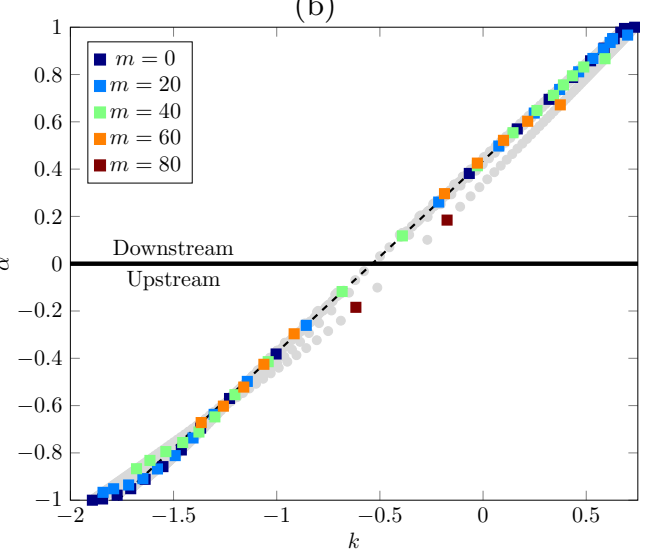

Figure 5: Plot of $k$ versus $\alpha$ for the realistic axial flow $M_{x}(r)$ from Figure 4a. The other parameters are $h=0.6558$ and (a) $\omega=20$ and (b) $\omega=80$. Selected values of $m$ have been highlighted with coloured squares. In black (dashed) is the relationship between $k$ and $\alpha$ for uniform flow $\overline{M_{x}}=0.445$, corresponding to the radial average. 
from the uniform axial flow case and hence establish the usefulness of cut-on ratio. However, the deviation is clearly linked to the degree of variation in the flow profile with radius. More work is needed to understand the sensitivity of $k(\alpha)$ to flow profile.

\subsubsection{Radially varying axial and swirling flow}

We now consider the most general case of radially varying axial flow and swirling flow representative of, for example, the interstage region of an aeroengine. To understand the general effects of swirl on the behaviour of $k(\alpha)$ it is instructive to consider the idealised case of solid body swirl of $M_{\theta}(r)=\Omega r$ with uniform axial flow. We initially use an idealised swirl model where the effect of swirl is simply to shift the frequency in (2) by a Doppler factor $-m \Omega$, as in (author?) [13] (so ignoring the effect of the mean swirl on the mean flow speed of sound and density in (A.2) and (A.3)). The $k(\alpha)$ relationship from (2) therefore becomes

$$
k_{m n}=\frac{\alpha_{m n}-M_{x}}{1-M_{x}^{2}} \frac{\omega-m \Omega}{\omega} .
$$

Thus, there is no longer a relation involving only $k$ and $\alpha$ since now we have an additional dependence on $m$.

We now investigate the precise dependence of $k$ on $\alpha$ for a flow profile comprising the realistic axial flow profile $M_{x}(r)$ plotted in Figure 4a, combined with four different idealised swirling flow profiles with increasing levels of swirl. These are given by $M_{\theta}(r)=0.3 r$ (solid body swirl), $M_{\theta}(r)=0.2 r+0.2, M_{\theta}(r)=0.1 r+0.4$ and $M_{\theta}(r)=0.6$, and are plotted in Figure 4b. The computed variation of $k$ with $\alpha$ is plotted in Figure 6 at a frequency of $\omega=20$. Note that the effects on the mean sound speed and density by the swirling flow is accounted for in these computations.

The variation of $k$ with $\alpha$ in these figures show similar features as described in Section 2.2.2 for the non-swirling flow but with some important differences. The most notable difference is that the relationship is no longer symmetric in $\pm m$. As a result, for a fixed radial mode order $n$, the trajectories that were approximately lines in Figure 3 now become loops. Each loop corresponds to a particular radial mode order, as explicitly labelled in Figure 6d (with $n=1$ corresponding to the most cut-on mode).

Finally we note that the modes associated with circumferential mode order $m=0$ lie very close to the black (dashed) line denoting the relationship between $k$ and $\alpha$ in uniform flow. This behaviour is readily understood from (8) for the case of solid body swirl with the idealised swirl model which predicts that swirl has no effect for these axi-symmetric modes. Therefore, the behaviour at $m=0$ closely mirrors Figure $5 \mathrm{a}$ which has the same axial flow profile but no swirl.

\subsection{Mode distribution function for cut-on ratio}

One of the advantages of representing modes by their cut-on ratio rather than mode indices $(m, n)$ is in the calculation of multi-mode sound field in the high frequency limit $[12,1]$. In these calculations the double summation over $m$ and $n$ is replaced by a single integration over cut-on ratio $\alpha$, where $\alpha$ is treated as a continuous variable on the domain $[-1,1]$. Central to these calculations is therefore knowledge of how the values of $\alpha$ are distributed, which is expressed as a probability density function $\eta(\alpha)$.

One such example of this approach is in a new measurement procedure, referred to here as the twomicrophone method [9], which allows the mode amplitude distribution to be deduced as a function of $\alpha$ from measurements of the coherence function between two acoustic acoustic measurements made at the duct wall. However, this method based on $\alpha$ is restricted to a uniform axial mean flow. This procedure will be discussed in some detail in Section 3 and a revised formulation based on wavenumber $k$ is presented subsequently that is valid for any arbitrary flow profile.

In a hollow duct with uniform axial flow, in the high-frequency limit, work by (author?) $[9,12,1]$ have shown analytically that $\alpha$ has the following distribution

$$
\eta(\alpha)=|\alpha|, \quad-1 \leq \alpha \leq 1
$$

This result predicts that there are comparatively very few modes close to $\alpha=0$ near cut-off, and most of the modes are clustered near the end points at $\alpha= \pm 1$, corresponding to well cut-on modes. 
(a)

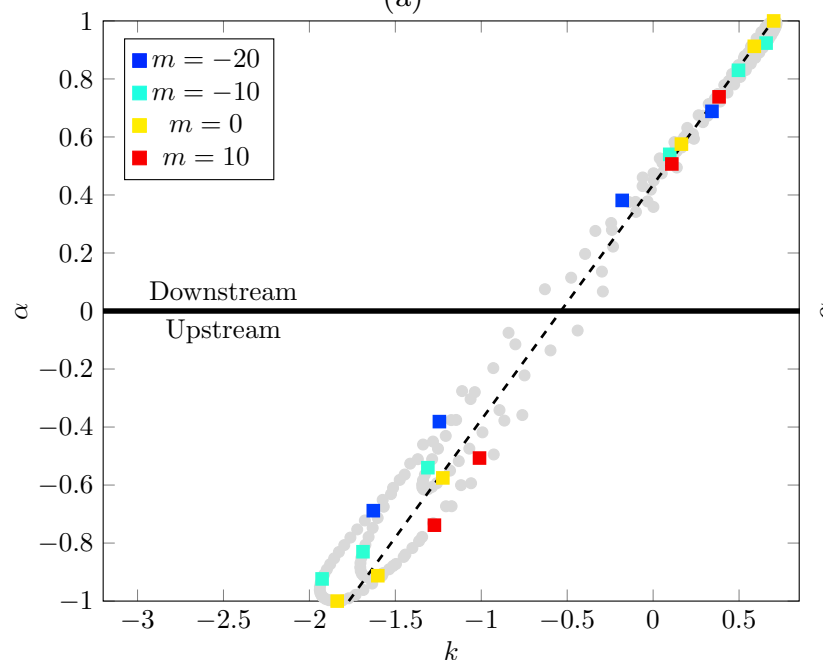

(c)

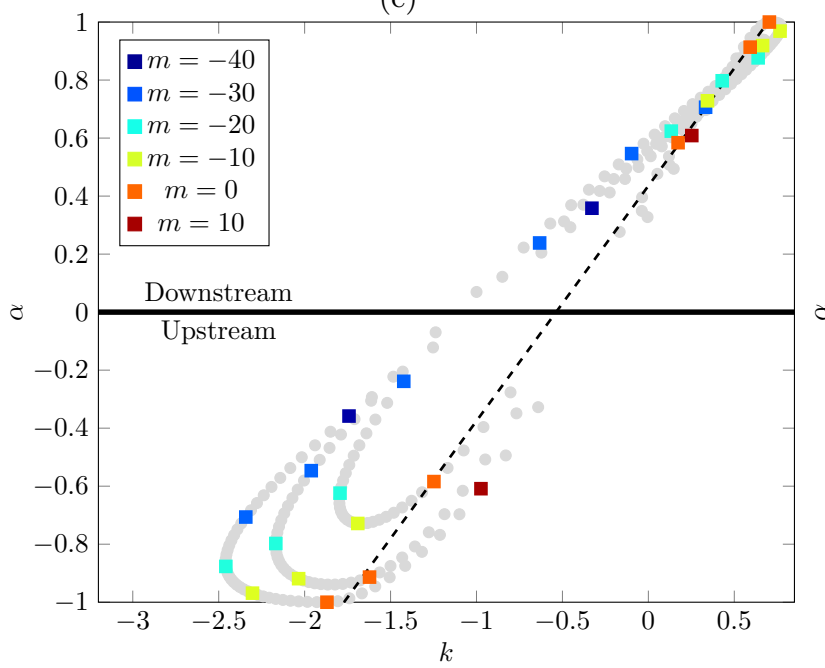

(b)

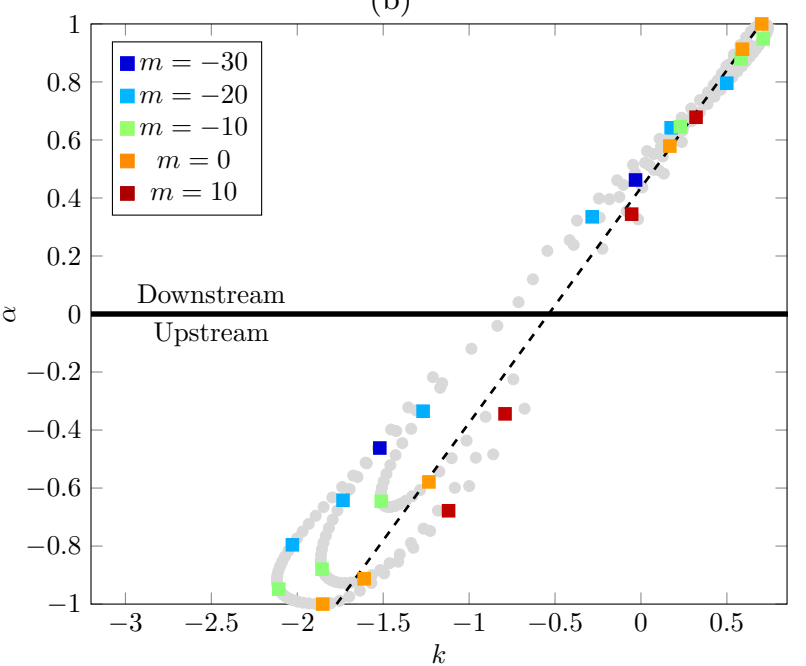

(d)

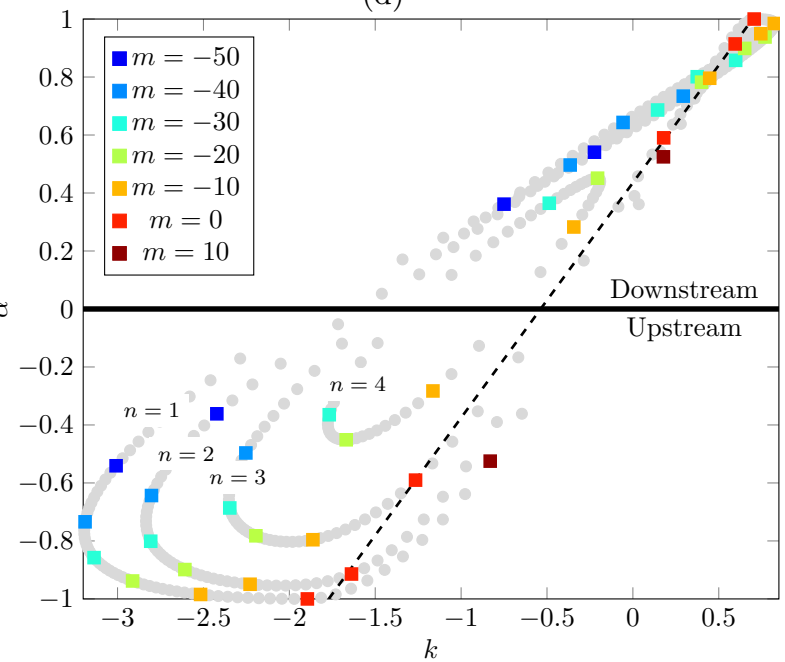

Figure 6: Plot of $k$ versus $\alpha$ (grey circles) for the axial flow from Figure 4 a and different swirling flows (a) $M_{\theta}(r)=0.3 r$; (b) $M_{\theta}(r)=0.2 r+0.2 ;$ (c) $M_{\theta}(r)=0.1 r+0.4$ and (d) $M_{\theta}(r)=0.6$. The other parameters are $h=0.6558$ and $\omega=20$. Selected values of $m$ have been highlighted with coloured squares. In black (dashed) is the relationship between $k$ and $\alpha$ for uniform axial flow $\overline{M_{x}}=0.445$ and no swirl.

The density function $\eta(\alpha)$ is now computed numerically for the case of uniform axial flow in an annular duct with $h=0.5$ and also the more general swirling flow $M_{x}(r)=0.3+0.2 r^{2}, M_{\theta}(r)=0.2 r+0.3$, also with $h=0.5$. These density functions are plotted in Figure $7 \mathrm{a}$ and Figure $7 \mathrm{~b}$ respectively, in blue (solid). The dashed red curve in this figure corresponds to the distribution of (9). Even for swirling flow, the distribution $\eta(\alpha)$ is observed to closely follow $|\alpha|$. These results clearly suggests that the definition of $\alpha$ in (7) appears to preserve the essential density function derived for uniform axial flow, irrespective of flow profile. The reasons for this unexpected result are currently not clear.

\subsection{Mode distribution for wavenumber}

So far in this section we have considered the relationship between wavenumber $k$ and cut-on ratio $\alpha$, and also the probability density function of $\alpha$. We therefore now turn our attention to the probability density function $\eta(k)$, which will be of use in Section 3 in a revised two-microphone method based on $k$. Note that unlike the $\eta(\alpha)$ distribution in Figure 7, the mode distribution is expected to differ for each flow profile. 
(a)

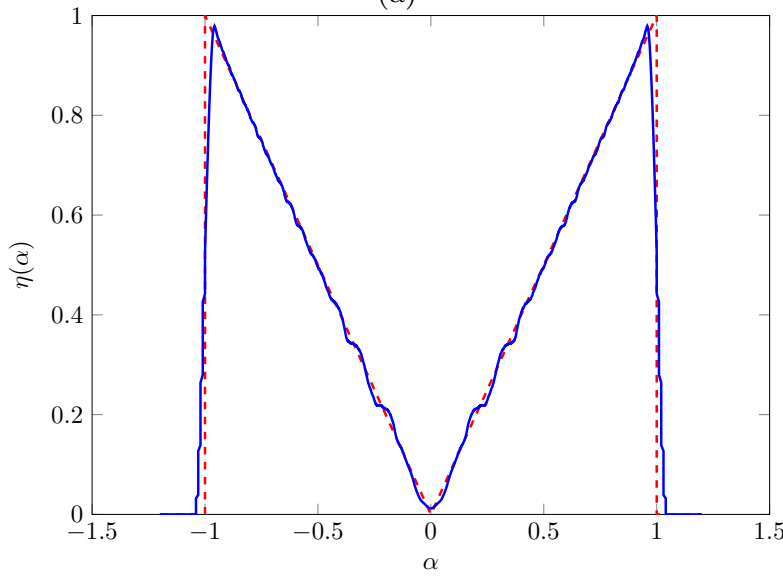

(b)

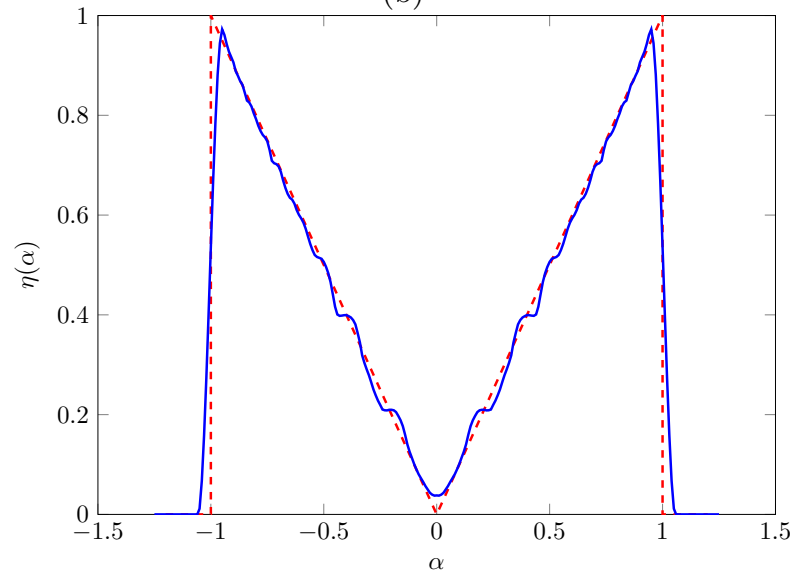

Figure 7: Plot of $\eta(\alpha)$ (in blue, solid) after calculating $\alpha$ for all cut-on modes for two different flows in an annular duct with $0.5<r<1$. We have plotted $|\alpha|$ in red (dashed). The flow parameters are (a) $M_{x}=0.5, M_{\theta}=0, \omega=150 ;(\mathrm{b})$ $M_{x}(r)=0.3+0.2 r^{2}, M_{\theta}(r)=0.2 r+0.3, \omega=80$.

\subsubsection{Uniform axial flow}

In the case of uniform axial flow, (2) indicates a linear relationship between $k$ and $\alpha$ from which $k$ takes values in the range

$$
-\frac{1}{1-M_{x}}<k<\frac{1}{1+M_{x}}
$$

for propagating modes, so, unlike $\alpha$ is not symmetric unless $M_{x}=0$. Substituting for $k$ in the probability density function for $\alpha$ in (9) and normalising $\eta(k)$ such that

$$
\int_{-1 /\left(1-M_{x}\right)}^{1 /\left(1+M_{x}\right)} \eta(k) \mathrm{d} k=1
$$

gives

$$
\eta(k)=\left(1-M_{x}^{2}\right)^{2}\left|k+\frac{M_{x}}{1-M_{x}^{2}}\right| .
$$

It is clear that $k=-M_{x} /\left(1-M_{x}^{2}\right)$ corresponds to $\alpha=0$ for modes at cut-off, with both having probability zero. The maximum value of the probability density function occurs for the most upstream and downstream cut-on modes, $k=-1 /\left(1-M_{x}\right)$ and $k=1 /\left(1+M_{x}\right)$ respectively, where $\eta(k)=1-M_{x}^{2}$.

\subsubsection{Varying swirling flow}

One of the advantages of expressing the distribution of modes according to their $\alpha$ values is that $\alpha$ across the range of propagating modes varies between -1 and 1 . In the case of uniform axial mean flow, the corresponding range of $k$ is readily determined from (10). However, in the general case of arbitrary radially varying swirling flow, the range of $k$ must be determined numerically, which we now show varies with frequency.

Table 1 lists the calculated values of the minimum and maximum values of $k$ for the range of propagating modes at different frequencies for the radially varying non-swirl case, $M_{x}(r)=0.4+0.4(r-h), M_{\theta}=0$ considered previously in Section 2.2.2. Table 1 makes clear the frequency dependence of the minimum and

\begin{tabular}{|c|r|r|r|r|r|}
\hline$\omega$ & 10 & 20 & 40 & 80 & 160 \\
\hline$k_{\min }$ & -1.7896 & -1.8382 & -1.8788 & -1.9060 & -1.9188 \\
$k_{\max }$ & 0.6987 & 0.7013 & 0.7135 & 0.7351 & 0.7523 \\
\hline
\end{tabular}

Table 1: Calculation of $k_{\min }$ and $k_{\max }$ for the swirling flow $M_{x}(r)=0.4+0.4(r-h), M_{\theta}=0$ in a duct $0.5<r<1$ at different frequencies, showing the weak frequency dependency. 
(a)

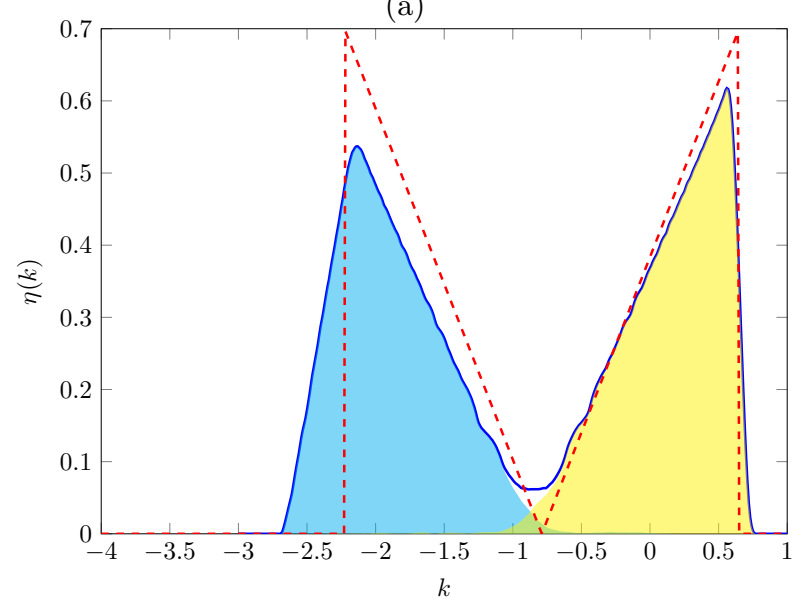

(b)

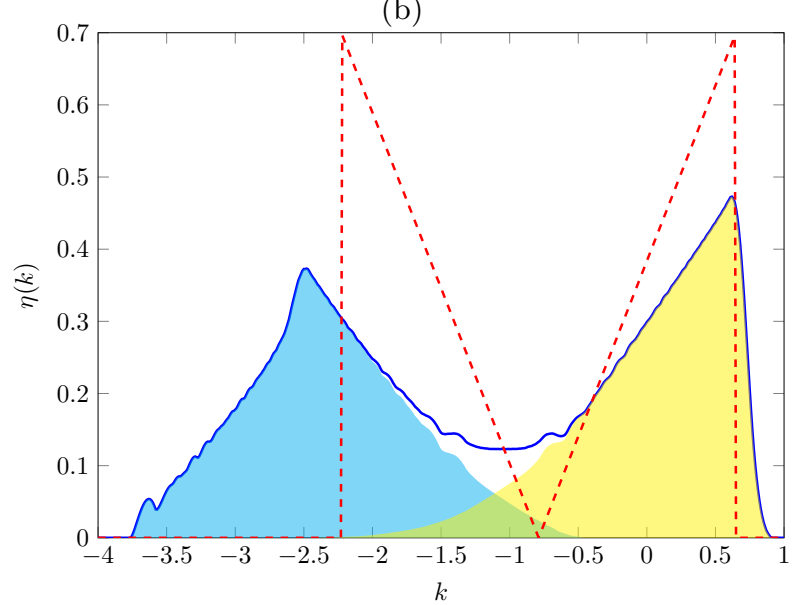

Figure 8: Plot of $\eta(k)$ (in blue, solid) after calculating $k$ for all cut-on modes for axial flow $M_{x}(r)=0.4+0.2 r$ in a duct $0.5<r<1$ at a frequency $\omega=150$. The swirl is (a) $M_{\theta}(r)=0.1 r+0.1 / r$ and (b) $M_{\theta}(r)=0.3 r+0.2 / r$. In blue is the distribution of upstream cut-on modes, while in yellow is the distribution of downstream modes. In red (dashed) we have plotted the density function $\eta(k)$ for the uniform axial flow $\overline{M_{x}}$, given by $(12)$.

maximum values of $k$ needed to quantify the range of propagating modes. However, as we let $\omega \rightarrow \infty$ then $k_{\min }$ and $k_{\text {max }}$ appears to converge to well defined limits. A similar study in swirling flow suggests that the observation is also valid in this case.

We now investigate the behaviour of the probability density function for an arbitrary swirling flow, obtained after numerically computing $k$ for all the propagating modes. In practice $\omega \gtrsim 100$ was found to be sufficiently high to obtain a smooth estimate for the density function $\eta(k)$.

The probability density functions $\eta(k)$ are compared in Figure 8 for two cases of identical axial flow profile $M_{x}(r)=0.4+0.2 r$ but with low (Figure 8a) and high (Figure 8b) levels of swirl, gives by the profiles $M_{\theta}(r)=0.1 r+0.1 / r$ and $M_{\theta}(r)=0.3 r+0.2 / r$. The radially averaged levels of swirl for the two cases are $\overline{M_{\theta}}=0.2136$ and $\overline{M_{\theta}}=0.5023$ respectively. The distributions were calculated at the non-dimensional frequency of $\omega=150$. The distribution of upstream propagating modes is depicted in yellow and the downstream modes in blue. For comparison, the density function of (12) for the uniform axial mean flow is plotted as a red dashed line.

The density functions $\eta(k)$ for swirling flow in Figure 8 exhibit several features distinct from the $\alpha$ distributions plotted in Figure 7 . The most striking observation is that $\eta(k)$ differs significantly from the case of the uniform axial flow in terms of shape, height and end points $k_{\min }$ and $k_{\max }$. As we increase the swirl velocity, these differences becomes more exaggerated, as seen in Figure 8b. One particularly noticeable feature in Figure 8 is the presence of an overlap region (in green) of $k$ in which upstream and downstream propagating modes share the same value of $k$. This phenomenon is also observed in flow profiles in which swirl is absent. For higher values of swirl, this effect is more pronounced, as seen in Figure 8b. However, this non-uniqueness is not a problem when either the upstream or downstream going modes are dominant, such as in an aeroengine duct inlet or bypass section.

By way of summary, therefore, a simple distribution for $\alpha$ with $\eta(\alpha)=|\alpha|$ for $-1 \leq \alpha \leq 1$ is observed for all swirling flows considered. However, due to the complex inter-relationship between the wavenumber and cut-on ratio, as shown in Section 2.2, the resulting density function in $k$ is complicated, depends on the flow profile and must be numerically determined.

\section{A new two-microphone method}

In this section we investigate a relatively new method for determining the mode amplitude distribution in which the concept of cut-on ratio was central. It is based on previous work by (author?) [9] in which the mode amplitude distribution may be determined as a function of cut-on ratio $\alpha$ from measurements of 
the coherence function between the acoustic pressures at two closely spaced microphones at the duct wall. Central to the formulation of the method is (2) linking $k$ to $\alpha$ for a uniform mean axial flow. The formulation in (author?) [9] is therefore restricted to the idealised case of uniform mean flow.

In this section we provide an alternative formulation in terms of the axial mode wavenumber $k$ which circumvents the need to invoke the relationship of (2) for uniform mean flow. The new formulation in terms of $k$ is therefore completely general, and therefore allows the mode amplitude distribution to be determined as a function of $k$ for any arbitrary flow profile, including swirl. This new formulation also highlights the deficiency of the cut-on ratio in arbitrary flows. We will show in the next section that the $\alpha$-based and $k$-based formulations give essentially equivalent mode amplitude distribution results for the flow profiles for which $k(\alpha)$ matches most closely the theoretical relationship of (2) for a uniform mean axial flow.

\subsection{Outline of method}

In this section the basic steps involved in the derivation of the expression for the mode amplitude distribution in terms of the pressure coherence function are presented. However, unlike in (author?) [9] we avoid the use of (2) linking $k$ and $\alpha$ which is valid only for uniform mean flow.

We begin by calculating the pressure cross spectrum (density) between two points separated by a distance $\Delta x$ at the same circumferential location on the outer duct wall. Making the assumption of uncorrelated mode amplitudes, the cross spectrum between the acoustic pressures at the outer wall of the duct with arbitrary $\theta$ and an axial separation distance of $\Delta x$ is given by

$$
S_{12}(\omega)=\mathbb{E}\left[\frac{p\left(1, \theta, x_{1}\right) p^{\star}\left(1, \theta, x_{1}+\Delta x\right)}{T}\right] .
$$

In this expression, $T$ is the time interval over which the Fast Fourier Transform of $p$ is taken, and $p$ is given in (1). Substituting (1) in (13) then gives the cross spectrum as

$$
S_{12}(\omega)=\sum_{m, n}\left[\mathbb{E}\left(\left|A_{m n}^{+}(\omega)\right|^{2}\right) e^{\mathrm{i} k_{m n}^{+} \Delta x}\left|P_{m n}^{+}(1)\right|^{2}+\mathbb{E}\left(\left|A_{m n}^{-}(\omega)\right|^{2}\right) e^{\mathrm{i} k_{m n}^{-} \Delta x}\left|P_{m n}^{-}(1)\right|^{2}\right],
$$

where we have separated the cross spectrum into downstream $(+)$ and upstream contributions $(-)$. The eigenfunctions (or mode shapes) $P_{m n}^{ \pm}(r)$ have been normalised such that

$$
\frac{2}{\left(1-h^{2}\right)} \int_{h}^{1}\left|P_{m n}^{ \pm}(r)\right|^{2} r \mathrm{~d} r=1 .
$$

The main assumption underlying the technique is that the relative mode amplitude distribution is only a function of the non-dimensional axial wavenumber $k_{m n}$. The mode amplitude distribution has been demonstrated to be solely a function of $\alpha[12,1]$ in uniform flow for a broad range of physically realistic source distributions. It must therefore be solely a function of $k$ in a uniform mean axial flow since $k$ and $\alpha$ are linearly related through (2). In this new formulation we will assume that the relative mode amplitude distribution only depends on $k$ for all flow profiles. The mode amplitudes may therefore be written in the form

$$
\mathbb{E}\left(\left|A_{m n}^{ \pm}(\omega)\right|^{2}\right)=S(\omega) \overline{a_{ \pm}^{2}}\left(k_{m n}^{ \pm}\right)
$$

where $S(\omega)$ is a frequency-dependent source term whose interpretation will be made clear below and $\overline{a_{ \pm}^{2}}$ describes the non-dimensional mode amplitude function of modal axial wavenumbers $k_{m n}^{ \pm}$, which is to be determined.

In the high-frequency limit (non-dimensional frequency $\omega \gtrsim 10)$ we can treat $\overline{a^{2}}(k)$ as a continuous variable. The discrete summation in (14) can therefore be replaced by an integration over $k$ that includes the density function $\eta(k)$ to account for the distribution of modes across their $k$-values, as plotted in Figure 8 and discussed in Section 2.4. Finally, we replace the square of the radial eigenfunctions $P_{m n}^{ \pm}(1)$ by their value at the duct wall (see (author?) [12]) averaged over all indices $m$ and $n$, given by

$$
\left\langle\left|P_{m n}^{ \pm}(1)\right|^{2}\right\rangle=2
$$


This approximation gives the largest error for modes with large $m$ values [9], but since there are relatively few of these modes the approximation should produce "negligible error" compared to the exact calculation.

After making these three approximations and noting that the number of modes with $k$ values between $k$ and $k+\mathrm{d} k$ is $N_{\omega} \eta(k) \mathrm{d} k$, where $N_{\omega}$ is the total number of propagating modes at the frequency $\omega$, we therefore arrive at the pressure cross spectrum at the duct wall:

$$
S_{12}(\widehat{\omega})=2 N_{\omega} S(\widehat{\omega}) \int_{k_{\min }}^{k_{\max }} \overline{a^{2}}(k) \eta(k) e^{\mathrm{i} \widehat{\omega} k} \mathrm{~d} k \text {, where } \widehat{\omega}=\omega \Delta x .
$$

Finally, we choose the non-dimensional mode amplitude distribution to be normalised such that

$$
\int_{k_{\min }}^{k_{\max }} \overline{a^{2}}(k) \eta(k) \mathrm{d} k=1 .
$$

Setting $\Delta x=0$ in (18) gives the autospectrum $S_{11}(\widehat{\omega})$ of pressure at the duct wall. Further noting the normalisation property of (19) defines the source term $S(\omega)$ in terms of the autospectrum of the pressure at the duct wall,

$$
S_{11}(\widehat{\omega})=2 N_{\omega} S(\widehat{\omega}) .
$$

We then approximate the pressure power spectral density at $x_{2}=x_{1}+\Delta x$, by $S_{22}(\widehat{\omega}) \approx S_{11}(\widehat{\omega})$ and introduce the complex coherence function $\psi_{12}(\widehat{\omega})$, which is defined at the duct wall and is given by

$$
\psi_{12}(\widehat{\omega})=\frac{S_{12}(\widehat{\omega})}{\sqrt{S_{11}(\widehat{\omega}) S_{22}(\widehat{\omega})}} .
$$

Finally, we arrive at an integral relationship between the complex coherence function $\psi_{12}(\widehat{\omega})$ and the mode amplitude distribution function $\overline{a^{2}}(k)$,

$$
\psi_{12}(\widehat{\omega})=\int_{k_{\min }}^{k_{\max }} \overline{a^{2}}(k) \eta(k) e^{\mathrm{i} \widehat{\omega} k} \mathrm{~d} k .
$$

This integral can be thought of as an integral over $\mathbb{R}$, since $\eta(k)=0$ for $k<k_{\min }$ or $k>k_{\max }$. The result is a Fourier transform relationship between the coherence function $\psi_{12}(\widehat{\omega})$ and the mode amplitude distribution $\overline{a^{2}}(k)$, which can be readily inverted to deduce $\overline{a^{2}}(k)$ in terms of the easily measurable quantity $\psi_{12}(\widehat{\omega})$ :

$$
\overline{a^{2}}(k)=\frac{1}{2 \pi \eta(k)} \int_{-\infty}^{\infty} \psi_{12}(\widehat{\omega}) e^{-i \widehat{\omega} k} \mathrm{~d} \widehat{\omega} \text { for } k_{\min }<k<k_{\max } .
$$

In the original formulation of this method [9] the additional step was taken of expressing $k$ in terms of $\alpha$ using (2) for the case of uniform mean flow to arrive at a Fourier Transform relationship between the mode amplitude distribution as a function of $\alpha$ and the coherence function. The original formulation in terms of $\alpha$ may be recovered from the general result of (23) by making the substitution $k=\left(\alpha-M_{x}\right) /\left(1-M_{x}^{2}\right)$ to give

$$
\overline{a^{2}}(\alpha)=\frac{1}{2 \pi|\alpha|} \int_{-\infty}^{\infty} e^{\mathrm{i} M_{x} \widehat{\omega}} \psi_{12}(\widehat{\omega}) e^{-\mathrm{i} \widehat{\omega} \alpha} \mathrm{d} \widehat{\omega} \text { for }-1<\alpha<1 \text {, where } \widehat{\omega}=\frac{\omega \Delta x}{1-M_{x}^{2}} .
$$

However, the current analysis demonstrates that this additional step is unnecessary in the case of uniform mean flow and incorrect for realistic flow profiles since there is no longer a linear relationship between $k$ and $\alpha$. The analysis above has demonstrated that the essential Fourier Transform relation between $\overline{a^{2}}$ and the coherence function is with the modal axial wavenumber $k$ and that further expressing it in terms of $\alpha$ is unnecessary and potentially inaccurate, as demonstrated in the simulations presented below in Section 4 . 


\subsection{Limitations of the new two-microphone method}

The previous section has demonstrated how the two-microphone method may be completely generalised to any arbitrary flow profile to allow the mode amplitude distribution to be determined as a function of $k$ based on pressure measurements at just two microphones at the duct wall. However, the formulation introduces a number of potential difficulties that must be considered that do not exist in the original formulation in terms of $\alpha$ for uniform mean flow.

One of the major difficulties with the use of the new formulation is that, whilst it can provide an accurate estimate for $\overline{a^{2}}$ in terms of $k$, the interpretation of $k$ in terms of modal propagation and radiation angles, $\varphi_{x}$ and $\psi_{P}$ respectively, is much less clear. This difficulty is an artefact of the radially varying flow and the difficulty in defining meaningful propagation and radiation angles in these flow profiles, rather than the new technique itself.

The second fundamental difficulty with the new formulation is the non-uniqueness of $k$ in describing upstream and downstream propagating modes. A small range of $k$ values have been shown to exist that correspond to both upstream and downstream going modes for different values of $m$, as seen in Figure 8 . Clearly, this non-uniqueness issue only presents difficulties when upstream and downstream going modes of roughly the same amplitude are present simultaneously.

Lastly, the new method requires an estimate for the probability density function $\eta(k)$, which we have shown in Section 2.4 varies considerably depending upon the mean flow profile. By contrast, the density function for $\alpha$, based on a limited number of simulations with different profiles, appears to be robust and given by $\eta(\alpha)=|\alpha|$. The new formulation therefore introduces extra computation time, but is not excessive if the range of flow profiles under consideration is limited.

\section{Evaluation of the two-microphone methods}

In this section, the accuracy of the original two-microphone method of (24) based on the cut-on ratio and the assumption of uniform mean flow is now compared against the new formulation of (23) based on $k$ in which no assumption is made about the nature of the mean flow profile. Their relative ability to deduce the mode amplitude distribution from the acoustic pressure coherence function is now compared. An idealised source distribution is assumed in this study comprising incoherent point monopole sources uniformly distributed over the duct cross section.

Four different swirling flow profiles are investigated for numerical validation of the two methods. The first is the axial flow profile shown in Figure 4a, which was measured in a scale-model test rig with hubto-tip ratio of $h=0.6558$, and the second is for the axial flow $M_{x}(r)=0.25+(r-h)$ with $h=0.5$. Both flow profiles have no swirling component. The third and fourth cases involve swirling flow, with the third case corresponding to the solid body swirl profile $M_{\theta}=0.3 r$ and the fourth case the swirling flow profile $M_{\theta}=0.1 r+0.4$, both shown in Figure 4b. In the last two cases the hub-to-tip ratio was $h=0.6558$ and the axial flow profile was assumed to be that of Figure $4 \mathrm{a}$.

The coherence function was deduced from the acoustic pressure cross spectrum of (14) with an assumed mode amplitude distribution function versus $\alpha$ corresponding to incoherent point monopole sources (in uniform axial flow with no swirl) uniformly distributed over the duct cross section. This is given by [12]

$$
\left|A_{m n}\left(\alpha_{m n}\right)\right|^{2} \propto \frac{1}{\alpha_{m n}^{2}} \frac{\left(1-\alpha_{m n} M_{x}\right)^{2}}{\left(1-M_{x}^{2}\right)^{2}},
$$

where $\left|A_{m n}^{+}\left(\alpha_{m n}\right)\right|^{2}=\left|A_{m n}\left(\alpha_{m n}\right)\right|^{2}$ for $\alpha_{m n}>0$ and $\left|A_{m n}^{-}\left(\alpha_{m n}\right)\right|^{2}=\left|A_{m n}\left(\alpha_{m n}\right)\right|^{2}$ for $\alpha_{m n}<0$.

In order to validate the new two-microphone formulation in terms of $k$, the mode amplitude distribution function must be known as a function of $k$. Substituting $k_{m n}=\left(\alpha_{m n}-M_{x}\right) / \beta^{2}$ for the case of uniform flow into (25) provides a theoretical mode distribution function in terms of $k$ that we wish to recover from the coherence function using (23), of the form

$$
\left|A_{m n}\left(k_{m n}\right)\right|^{2} \propto \frac{\left(1-k_{m n} M_{x}\right)^{2}}{\left(M_{x}+k_{m n}\left(1-M_{x}^{2}\right)\right)^{2}} .
$$


The mode amplitude distributions in terms of $\alpha$ and $k$ were used in (24) and (23) respectively to deduce the complex coherence function at 100 frequency points between $\omega=1$ to $\omega=101$ in equal increments of $\Delta \omega=1$. The coherence function was computed separately for upstream and downstream going modes for the two mode amplitude distribution functions of (25) and (26). The microphone non-dimensional axial separation distance was chosen to correspond to $\Delta x=0.2 \mathrm{~m}$ to ensure that the coherence function decays sufficiently to zero and hence achieve convergence of the integrations in (23) and (24). The coherence functions based on the distributions of $\alpha$ and $k$ were then used in (24) and (23) to deduce the mean square mode amplitude distribution as a function of $\alpha$ and $k$. The amplitude distribution functions obtained from this procedure are plotted in subsequent figures and compared against their theoretical distribution functions of $(25)$ and (26).

\subsection{Axial flow}

(a)

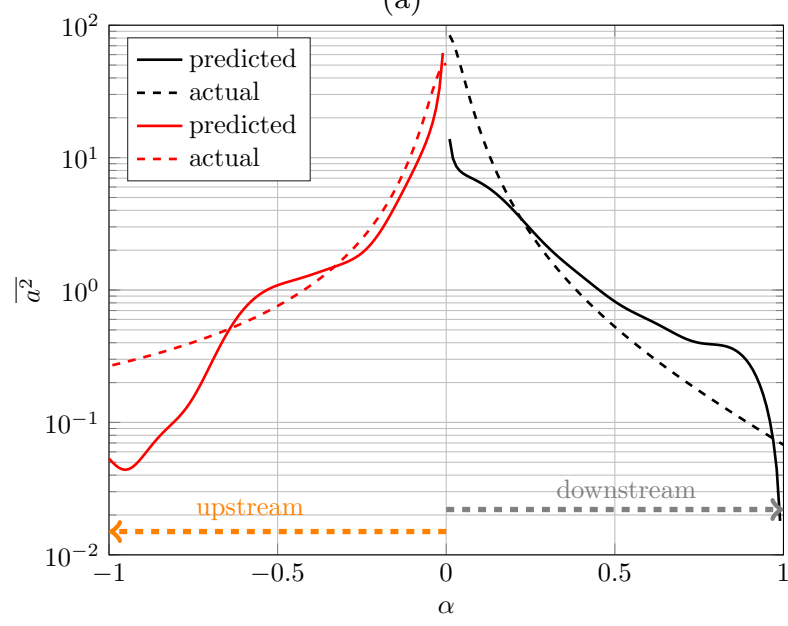

(b)

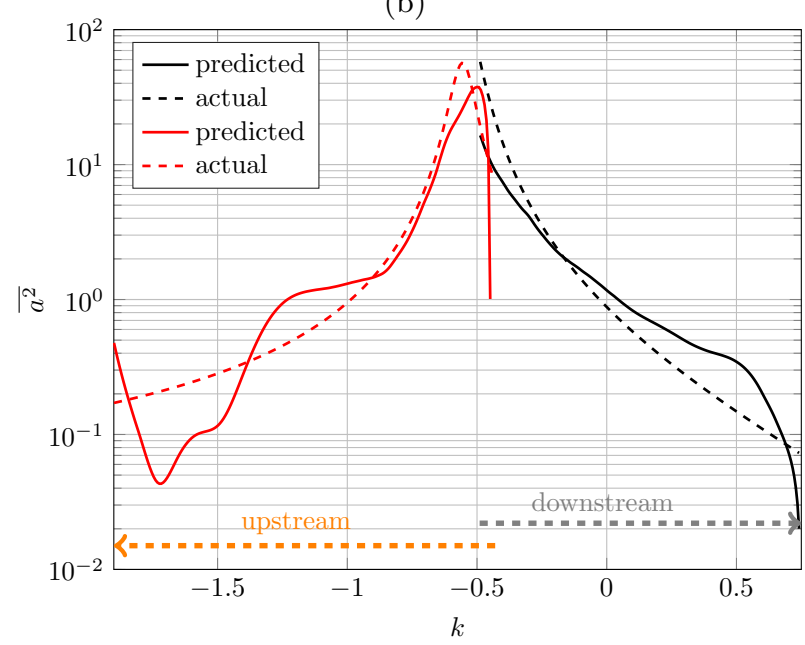

Figure 9: Prediction results for $\overline{a^{2}}$ for the axial flow shown in Figure 4a, from (author?) [15]. (a): $\alpha$-based method, (b): $k$-based method.

Figure 9 shows the comparison of the mode amplitude distribution deduced from the $\alpha$-based and $k$-based two-microphone methods for the purely axial flow profile measured in the bypass section of a scale-model rig (plotted in Figure 4a) with their corresponding theoretical distributions of (25) and (26) respectively.

Generally good agreement between the predicted and actual mode distribution functions is observed in both cases, with levels of error being similar in both the $\alpha$ and $k$-based methods. The greatest error (of more than $5 \mathrm{~dB}$ ) is found to occur for the extreme values of $\alpha$ and $k$. The range of $k$ corresponding to the upstream and downstream modes are represented by horizontal arrows. Note the small region of overlap in $k$ between about -0.5 and -0.45 , which represents both upstream and downstream going modes. Modes represented by their $k$ values in this region are therefore non-unique. This phenomenon represents one of the disadvantages of the $k$-based two-microphone method. We show below the region of overlap becomes greater when swirl is present, as we saw with the probability density function in Section 2.4. However, this non-uniqueness phenomenon is only an issue when both upstream and downstream propagation of similar amplitudes are present simultaneously.

We now consider the second case of a purely axial flow, $M_{x}(r)=0.25+(r-h)$. A comparison of the predicted and actual mode amplitude distribution functions for this profile are shown in Figures 10a and $10 \mathrm{~b}$ for the $\alpha$-based and $k$-based two-microphone methods respectively.

For this radial profile the $k$-based method provides consistently better agreement with the theoretical distribution, except at large negative $k$ values, corresponding to well cut-on modes whose corresponding $\alpha$ values are close to -1 . The range of $k$ for which upstream and downstream going modes overlap is also greater for this axial flow profile example. The generally poor performance of the $\alpha$-based method could have been predicted from the extent of deviation of $k(\alpha)$ from the uniform mean flow result of (2), as seen 
(a)

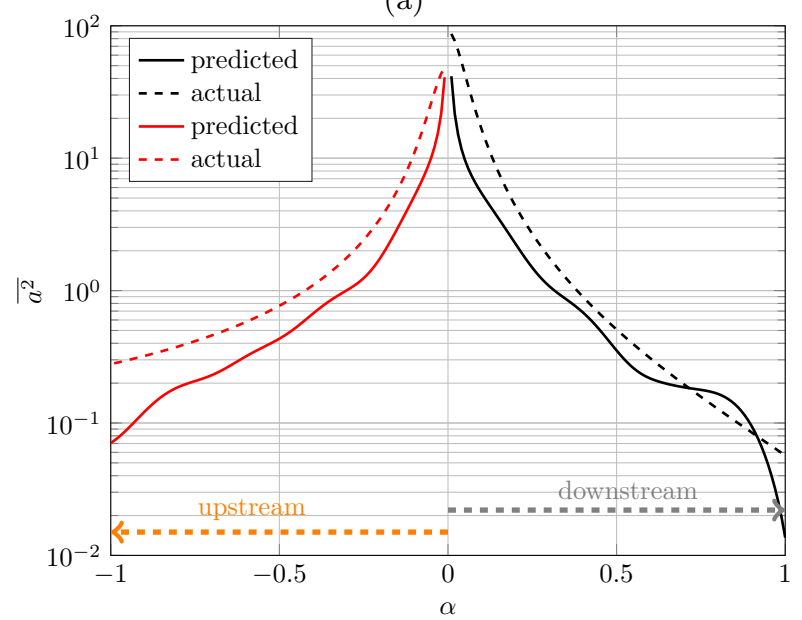

(c)

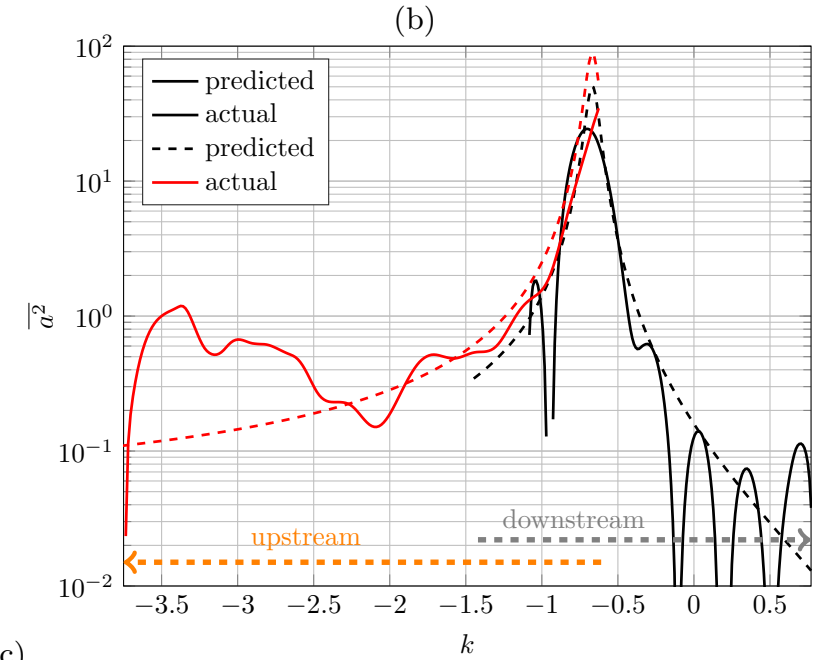

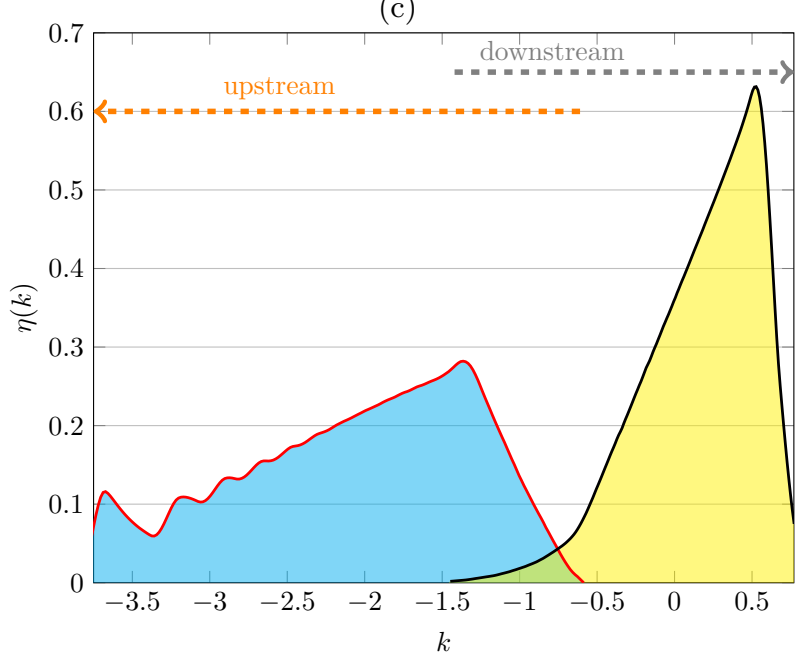

Figure 10: Prediction results for $\overline{a^{2}}$ for the axial flow of $M_{x}(r)=0.25+(r-h)$. (a): $\alpha$-based method, (b): $k$-based method. (c): mode density function $\eta(k)$.

in Figure 3c. For the previous realistic axial flow example, there was a much smaller deviation from (2), as seen in Figure 5b, which explains the better performance of the $\alpha$-based method in this example.

One reason for the poor performance of the two-microphone method in this example at large negative $k$ values is due to the poor estimate of the probability density function due to the relative sparseness of modes in this $k$ range, as shown in Figure 10c.

The density function $\eta(k)$ exhibits non-physical oscillatory behaviour for values of $k \lesssim-2.5$ corresponding to the range of $k$ in Figure 10b of poorest agreement with the theoretical distribution. To obtain a smoother, more accurate estimate for $\eta(k)$ in in this example, the propagating modes from all frequencies in the range $1 \leq \omega \leq 101$ were used to compute the distribution plotted in Figure 10c. This sparseness phenomenon is less of an issue for the $\alpha$-based method, since the probability density function appears to follow $\eta(\alpha)=|\alpha|$ for all flow profiles.

\subsection{Swirling flow}

We now consider the effect of swirl on the $\alpha$-based and $k$-based two-microphone methods. Figure 11 shows a comparison of the predicted and actual mode amplitude distribution functions for the case in which the axial flow profile $M_{x}(r)$ corresponds to the measured data in Figure 4a with a swirl component given by $M_{\theta}(r)=0.3 r$. 
(a)

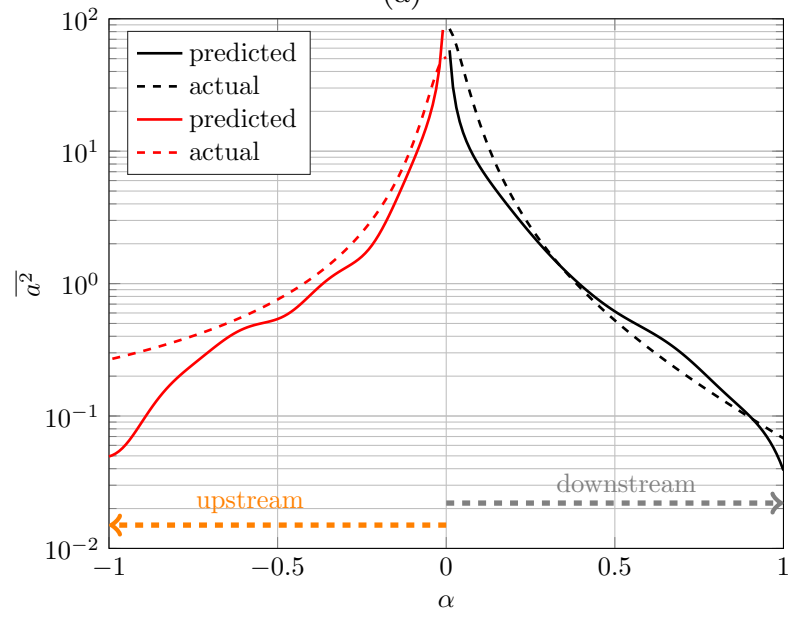

(b)

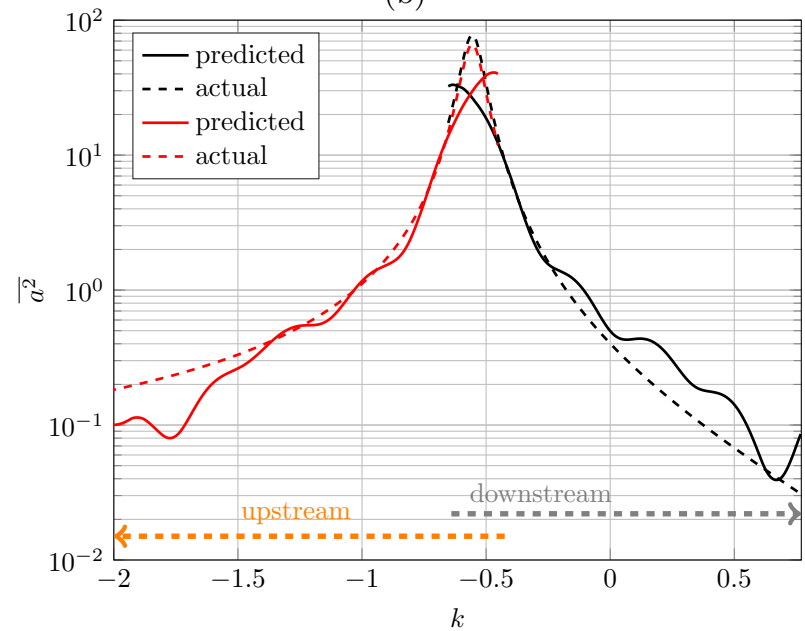

Figure 11: Prediction results for $\overline{a^{2}}$ for the axial flow shown in Figure 4a and solid body swirl of $M_{\theta}(r)=0.3 r$. (a): $\alpha$-based method, (b): $k$-based method.

Both methods can be seen to provide satisfactory agreement with the actual distribution but with the $k$ based method providing marginally better agreement overall. In this case the overlap between upstream and downstream propagating modes is relatively small. Again, this level of agreement with the $\alpha$-based method could have been anticipated from the generally good levels of agreement between the exact relationship $k(\alpha)$ and the uniform mean flow case, plotted in Figure 6a.

Finally, we examine the effect of increasing the level of swirl. Figure 12 shows a comparison of the mode distribution functions versus $\alpha$ and $k$ when the swirl velocity profile is increased to $M_{\theta}(r)=0.1 r+0.4$. The predicted mode amplitude distribution for the $\alpha$-based method is generally over-estimated by more than $5 \mathrm{~dB}$ over the full range of modes compared to the actual distribution. Generally good levels of agreement are established for the $k$-based method, except for large negative $k$ values owing to the relatively poor estimate of $\eta(k)$ deduced in this example from the modes at just the highest frequency $\omega=101$. Once again, the poor performance of the $\alpha$-based method in this example is consistent with the generally large deviation of $k(\alpha)$ from the theoretical relationship in (2) assumed in the method, shown in Figure 6c.

(a)

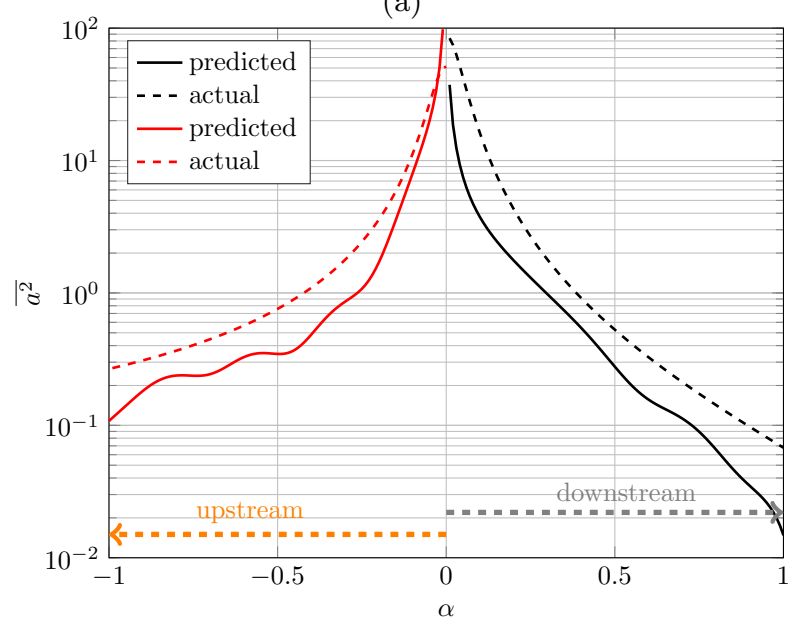

(b)

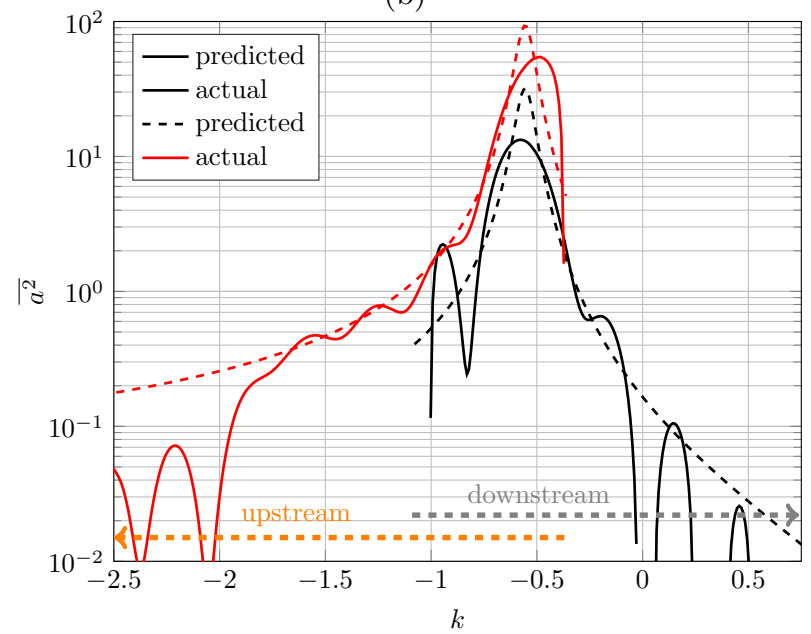

Figure 12: Prediction results for $\overline{a^{2}}$ for the axial flow shown in Figure 4a and swirl of $M_{\theta}(r)=0.1 r+0.4$. (a): $\alpha$-based method, (b): $k$-based method. 


\section{Discussion and Conclusions}

Definition of cut-on ratio in arbitrary flow profiles.

The concept of modal cut-on (or cut-off) ratio has been widely used as a non-dimensional measure of the degree to which an acoustic mode propagating along a duct is cut-on. In general terms, it represents how far in frequency a single mode is excited above its cut-on frequency. In the idealised case of a uniform mean axial flow its definition and its relation to the modal wavenumber components is clear and unambiguous, and can be used to deduce modal transmission and radiation angles. Equally importantly, the modal cut-on ratio $\alpha$ can be used in place of the usual modal indices $(m, n)$ to characterise modal propagation. However, the definition of cut-on ratio in the more realistic case in which the axial flow speed is radially varying, and which may also contain swirl, is not clear and is open to interpretation.

In the case of a uniform axial mean flow, the definition of cut-on ratio arises naturally as the ratio of axial and transverse modal wavenumber components (cf. (3)). This definition of cut-on ratio can in turn be expressed in terms of the excitation frequency relative to its cut-on frequency $\omega_{m n}$, as in (4). In the more general case of arbitrary flow profile there is no simple relation between $\alpha$ and the modal wavenumber components. However, in this paper we choose, arbitrarily, the identical expression of cut-on ratio

$$
\alpha_{m n}= \pm \sqrt{1-\left(\frac{\omega_{m n}}{\omega}\right)^{2}},
$$

for arbitrary flow profiles, where $\omega_{m n}$ must be computed numerically. This definition has the advantage of being consistent in all cases and, unexpectedly, appears to have the same probability density function $\eta(\alpha)$, irrespectively of flow profile. The reasons for this is currently unknown but provides some confidence in the choice of definition of $\alpha$. However, this paper has shown that this definition leads to difficulties in its interpretation and application. We found that in general the relationship between axial wavenumber and cut-on ratio is significantly more complicated in swirling flow.

Application to the two-microphone method for high frequency mode detection.

By way of illustrating the difficulty with defining a generalised cut-on ratio in an arbitrary flow profile, the accuracy of a new but previously studied measurement method (the two-microphone method) was investigated. The aim of the method was to express the mode amplitude distribution as a function of $\alpha$ from measurements of the coherence function between the acoustic pressures at two closely spaced microphones at the duct wall. Central to the validity of the measurement technique is the assumption that the relationship between $k$ and $\alpha$ for a uniform axial mean flow is accurate in all flow profiles. The two-microphone method was applied to predictions of the coherence function for four flow profiles based on an assumed mode amplitude distribution function of $\alpha$. Two of the profiles were purely radially varying axial flows while the other two involved different degrees of swirl. As expected, agreement between the recovered distributions and the exact distributions were poorest when the variation of $k$ with $\alpha$ deviated furthest from the relationship for a uniform axial mean flow.

This difficulty was circumvented by simply re-formulating the two-microphone method directly in terms of axial wavenumber $k$ without making any assumed dependence between $k$ and $\alpha$. This new formulation is therefore completely general and applies to any arbitrary flow profile. However, whilst this new formulation allow for the accurate determination of mode amplitude in any flow profile, it has been shown to have the following disadvantages:

1. The interpretation of $k$, and its relationship to modal propagation angles is complicated for arbitrary profiles and must generally be determined numerically.

2. The probability density function $\eta(k)$ must be determined by numerical computation whereas for $\alpha$ it appears to closely follow (9) irrespective of flow profile, even in the presence of swirl.

3. Modal axial wavenumbers $k$ are no longer unique for upstream and downstream going modes, with the size of the overlap region dependent on the flow profile. This is only an issue when upstream and downstream going modes of comparable amplitude are present simultaneously. 


\section{Acknowledgements}

All three authors were funded by and supported by the ENOVAL (ENgine mOdule VALidators) project, part of the Europeans Union's technology programme (grant number 604999). The authors would also like to thank Dr Brian Tester (ISVR) for providing the realistic flow data in Figure 4a.

\section{Appendix A. Calculating the eigenmodes in swirling flow}

We now discuss how to calculate the eigenmodes $k_{m n}$ and associated eigenfunction (or mode shape) $P_{m n}(r)$ for swirling, inviscid, homentropic flow in a duct. This is based upon the method in (author?) [16] and (author?) [17], which is outlined below. A cylindrical coordinate system is used, with $x$ the axial coordinate, $r$ the radial coordinate and $\theta$ the azimuthal coordinate, with $\underline{u}, \underline{v}$ and $\underline{w}$ being the velocity components in the $x, r$ and $\theta$ directions respectively. Given a base flow of the form

$$
\left(M_{x}(r), 0, M_{\theta}(r)\right),
$$

we calculate from the Euler equations and the gas thermodynamic relations that the base flow speed of sound is given by

$$
c_{0}^{2}(r)=1+(\gamma-1) \int_{1}^{r} \frac{M_{\theta}^{2}(s)}{s} \mathrm{~d} s,
$$

while the density is given by

$$
\rho_{0}(r)=\left[c_{0}^{2}(r)\right]^{1 /(\gamma-1)},
$$

and finally the pressure is given by

$$
p_{0}(r)=\frac{1}{\gamma}-\int_{r}^{1} \frac{\rho_{0}(s) M_{\theta}^{2}(s)}{s} \mathrm{~d} s
$$

where $\gamma$ is the ratio of specific heat capacities $(\gamma=1.4)$ for air. We then Fourier transform the standard mass, energy and momentum equations using

$$
\{u, v, w, p\}(r, x, \theta, t)=\int \sum_{m} \int\{U(r), V(r), W(r), P(r)\} e^{\mathrm{i} k x} \mathrm{~d} k e^{\mathrm{i} m \theta} e^{-\mathrm{i} \omega t} \mathrm{~d} \omega .
$$

After rearranging, the eigenvalues satisfy

$$
\begin{array}{r}
\frac{M_{x} \bar{\Lambda}}{c_{0}^{2} \zeta} \mathrm{i} U+\left[\frac{M_{x}}{c_{0}^{2} \zeta} \frac{\mathrm{d} M_{x}}{\mathrm{~d} r}-\frac{1}{r \zeta}-\frac{M_{\theta}^{2}}{\zeta r c_{0}^{2}}\right] V-\frac{1}{\zeta} \frac{\mathrm{d} V}{\mathrm{~d} r}-\frac{m}{r \zeta} \mathrm{i} W-\frac{\mathrm{i} \bar{\Lambda}}{c_{0}^{2} \rho_{0} \zeta} P=k \mathrm{i} U, \\
-\frac{\bar{\Lambda}}{M_{x}} V-\frac{2 M_{\theta}}{r M_{x}} \mathrm{i} W+\frac{\mathrm{i}}{\rho_{0} M_{x}} \frac{\mathrm{d} P}{\mathrm{~d} r}-\frac{\mathrm{i} M_{\theta}^{2}}{\rho_{0} M_{x} r c_{0}^{2}} P=k V, \\
-\frac{1}{M_{x}}\left[\frac{M_{\theta}}{r}+\frac{\mathrm{d} M_{\theta}}{\mathrm{d} r}\right] V-\frac{\bar{\Lambda}}{M_{x}} \mathrm{i} W-\frac{\mathrm{i} m}{r \rho_{0} M_{x}} P=k \mathrm{i} W, \\
-\frac{\rho_{0} \bar{\Lambda}}{\zeta} U+\mathrm{i} \frac{\rho_{0}}{\zeta}\left[\frac{\mathrm{d} M_{x}}{\mathrm{~d} r}-\left(1+\frac{M_{\theta}^{2}}{c_{0}^{2}}\right) \frac{M_{x}}{r}\right] V-\frac{\mathrm{i} \rho_{0} M_{x}}{\zeta} \frac{\mathrm{d} V}{\mathrm{~d} r}+\frac{m \rho_{0} M_{x}}{r \zeta} W+\frac{M_{x} \bar{\Lambda}}{c_{0}^{2} \zeta} P=k P,
\end{array}
$$

with boundary conditions

$$
V(h)=0 \text { and } V(1)=0,
$$

where

$$
\bar{\Lambda}=\frac{m M_{\theta}}{r}-\omega \text { and } \zeta=1-\frac{M_{x}^{2}}{c_{0}^{2}} .
$$

We solve this eigenvalue problem using Chebfun [18] in MATLAB, which avoids having to discretise the derivatives. This allows the calculation of $k_{m n}$ and $P_{m n}(r)$. Our numerical eigenmode solver was validated 
against the results from the program in (author?) [16]. We find that we get perfect agreement in all cases with (author?) [16] for a duct with hard walls.

If we want to take into account non-zero boundary layer thickness effects, we need to use a more complicated modified Myers boundary condition (rather than (A.6e)) to account for the thickness which was derived in (author?) [19].

\section{Appendix B. Numerically calculating the cut-on frequency}

The essential idea to calculate the cut-on frequency $\omega_{m n}$ is that we vary the frequency $\omega$ and solve the eigenvalue problem numerically for the eigenmodes $k_{m n}$ until the $n$-th upstream and downstream modes coalesce and become cut-off. The precise frequency where this happens will be denoted $\omega_{m n}$.

Given a fixed value of $\omega$ and an azimuthal number $m$, we can calculate all the eigenmodes $k_{m n}$ by solving the eigenvalue problem in (A.6). Let $k_{m n}^{\star}$ denote the cut-on eigenmodes. Assume that $n$ ranges from 1 to $N$. Let $\mathcal{N}$ denote the number of cut-on modes, so $\mathcal{N}(\omega)=N$.

We illustrate the procedure when $\omega=12, m=0, M_{x}=0.5$ and $M_{\theta}=0$, for which there are three cut-on modes (so $N=3$ ).

- Make an initial guess. We first try and find frequencies $\omega^{j}<\omega$ where we have $1,2, \ldots, N$ cut-on eigenmodes. For example, we could try guessing $\omega^{j}=j \omega / N$ has $j$ cut-on modes.

We calculate that $\omega^{1}=4, \omega^{2}=8, \omega^{3}=12$ and that $\mathcal{N}\left(\omega^{1}\right)=1, \mathcal{N}\left(\omega^{2}\right)=2$ and $\mathcal{N}\left(\omega^{3}\right)=3$.

- Refine guess. Suppose we find that frequencies $\omega^{j}$ and $\omega^{j+1}$ have the same number of cut-on modes $j$, but $\omega^{j+2}$ has $j+2$ cut-on modes. We refine the guess by letting $\omega^{j+1}=\left(\omega^{j+1}+\omega^{j+2}\right) / 2$ and hoping that for the new value of $\omega^{j+1}, \mathcal{N}\left(\omega^{j+1}\right)=j+1$. We carry on a similar procedure until we have $N$ different frequencies $\omega^{j}$, where $\mathcal{N}\left(\omega^{j}\right)=j$ for $j=1, \ldots, N$.

Our initial guess had $\mathcal{N}\left(\omega^{j}\right)=j$ for $j=1, \ldots, N$ so this is not required.

- Bounded regions. Let $\omega^{0}=0$. We have now deduced a range for the cut-on frequency for each index. For $1 \leq n \leq N$ we have that $\omega^{n-1}<\omega_{m n}<\omega^{n}$.

- Introduce distance function. For a given $\omega$, introduce the distance function $d(\omega)=\mid \min \left(k_{m n \text {,down }}^{\star}\right)-$ $\max \left(k_{m n, \text { up }}^{\star}\right) \mid$, which is the distance between the closest upstream and downstream mode. For example, we find that $d\left(\omega^{1}\right)=10.6667, d\left(\omega^{2}\right)=15.3989$ and $d\left(\omega^{3}\right)=13.1893$.

- Find zeros of the distance function. For the cut-on frequencies, we have $d\left(\omega_{m n}\right)=0$. In the range $\omega^{n-1}<\omega_{m n}<\omega^{n}$ we have precisely one zero, due to our definition of $\omega^{n}$. We use a numerical solver (eg Newton-Raphson) to solve $d\left(\omega_{m n}\right)<\varepsilon$ to a tolerance of $\varepsilon$, which takes $f(n, \varepsilon)$ iterations to converge.

We find that the three cut-on frequencies are $\omega_{0,1}=0, \omega_{0,2}=5.5372$ and $\omega_{0,3}=10.9346$

Since we have to solve the eigenvalue problem at least $N$ times to get the initial guesses $\omega^{j}$, and then $\sum_{j=1}^{N} f(j, \varepsilon)$ to find the cut-on frequencies, we can see that we have to solve the eigenvalue problem a significant amount of times. Thus, the method to find the cut-on frequency and hence the cut-on ratio from (4) will be very expensive.

One way to speed up the method would be to find the eigenmodes faster, which might be possible by reducing the required accuracy. Alternatively, we could try and investigate whether there is a method which can determine whether a matrix has repeated eigenvalues without solving for them. To the authors knowledge, there is no method for doing this at present.

\section{References}

[1] E. J. Rice. Multimodal far-field acoustic radiation pattern using mode cutoff ratio. AIAA Journal, 16(9):906-911, 1978.

$[2]$ N. K. Agarwal. The sound field in fully developed turbulent pipe flow due to internal flow separation, part 2: modal amplitude and cut-off frequencies. Journal of Sound and Vibration, 175(1):65-76, 1994. 
[3] W. Eversman. Broadband noise suppression for turbofan inlet applications. International Journal of Aeroacoustics, 15(4-5):367-394, 2016

[4] L. D. Koch. Predicted and measured modal sound power levels for a fan ingesting distorted inflow. In $16 t h A I A A / C E A S$ Aeroacoustics Conference, Stockholm, Sweden, 2010.

[5] M. Nallasamy. Computation of noise radiation from turbofans: A parametric study. NASA Contractor Report 198359, 1995.

[6] E. J. Rice, M. Heidmann, and T. Sofrin. Modal propagation angles in a cylindrical duct with flow and their relation to sound radiation. In 17th Aerospace Sciences Meeting, 1979.

[7] C. J. Chapman. Sound radiation from a cylindrical duct. Part 1. Ray structure of the duct modes and of the external field. Journal of Fluid Mechanics, 281:293-311, 1994.

[8] C. J. Chapman. Sound radiation from a cylindrical duct. Part 2. Source modelling, nil-shielding directions, and the open-to-ducted transfer function. Journal of Fluid Mechanics, 313:367-380, 1996.

[9] P. Joseph. A two-microphone method for the determination of the mode amplitude distribution in high-frequency ducted broadband sound fields. The Journal of the Acoustical Society of America, 142(4):2019-2029, 2017.

[10] E. J. Rice. Acoustic liner optimum impedance for spinning modes with mode cut-off ratio as the design criterion. In $3 r d$ AIAA Aeroacoustics Conference, Palo Alto (CA), USA, 1976.

[11] E. J. Rice. Inlet noise suppressor design method based upon the distribution of acoustic power with mode cutoff ratio. In Advances in Engineering Science, volume 3, pages 883-894, 1976.

[12] P. Joseph, C. L. Morfey, and C. R. Lowis. Multi-mode sound transmission in ducts with flow. Journal of Sound and Vibration, 264(3):523-544, 2003.

[13] C. L. Morfey. Sound transmission and generation in ducts with flow. Journal of Sound and Vibration, 14(1):37-55, 1971.

[14] M. Abramowitz and I. A. Stegun. Handbook of mathematical functions, with formulas, graphs, and mathematical tables. Dover books on advanced mathematics, tenth edition, 1972.

[15] B. J. Tester and P. B. Murray. An in-duct to far-field phased array technique for validation of fan broadband liner performance at representative engine Mach numbers. In 19th AIAA/CEAS Aeroacoustics Conference, Berlin, Germany, 2013.

[16] H. Posson and N. Peake. The acoustic analogy in an annular duct with swirling mean flow. Journal of Fluid Mechanics, 726:439-475, 2013.

[17] J. R. Mathews and N. Peake. The acoustic Green's function for swirling flow in a lined duct. Journal of Sound and Vibration, 395:294-316, 2017.

[18] T. A. Driscoll, N. Hale, and L. N. Trefethen. Chebfun Guide. Pafnuty Publications, Oxford, 2014.

[19] J. R. Mathews, V. Masson, S. Moreau, and H. Posson. The modified Myers boundary condition for swirling flow. Journal of Fluid Mechanics, 2017. Submitted. 